\title{
Chitosan nanoparticles: an emerging weapon against the cancer
}

\section{Editorial}

Cancer has the highest mortality rate in the world as compared to the other disorders mainly due to non availabity of proper treatment at advance stage. Current cancer treatment involves the radiation therapy and chemotherapy both of which exerts side effects in the cancer individual that lead to death of the majority of patients Extensive research work is being carried out in order to reduce the side effects of anticancer drugs. These side effects are mainly due to the non-specificity of drugs to the target tissues. Therefore there is a need to find out the drug delivery system which can efficiently kill the cancer cells without harming normal healthy cells.

Nanotechnology in Medicine offers several advantages over present methods of treatment for several diseases. One of the major applications of nanotechnology use in medicine is the use of nanoparticles to deliver drugs or other substances to target specific types of cells, mainly the cancer cells or cancer stem cells. Nanoparticles are designed in such a way that they will deliver drugs directly only to specific affected cells without harming normal cell types and therefore nanoparticles that deliver anticancer drugs directly to cancer cells are under investigation. Many of such nanoparticles are giving remarkable progress in making this in reality. ${ }^{1}$ According to Dr. Lawrence Tamarkin, who is present CEO of this company, CytImmune Sciences, a biopharmaceutical company in US has designed to treat cancer by using nanotechnology which reduces side effects and minimized treatment cost for cancer to improve the quality life of cancer patient Whereas, BIND Biosciences, Inc, an another biopharmaceutical company has used medicinal Nano engineering platform to develop best qualities of drugs to provide significant improvement in the treatment for various diseases and they have already successful in doing targeted therapy for cancer which is presently at Phase $1 \&$ Phase 2 clinical trials. Many scientists around the globe have tried to use this technology in the field of medical science. Nanoparticles are becoming more famous day by day because of their enormous application in the diagnostics and therapeutics formulation. ${ }^{2}$ In the recent advances of nanoparticle formation, variety of materials have been tried and tested for their medical values. The scientists have moved one step further by adapting encapsulation technology in the nanoparticles and selected potential carrier molecules which have shown their ability to carry a drug molecule and deliver it to the specific organ with a sustained release. This process resulted into killing of only cancer cells without harming normal cells of cancer patients. ${ }^{3}$

One of such carrier molecules which have emerged in a last decade is Chitosan, derived from chitin and which is intern obtained from shrimp. ${ }^{4}$ Variety of methods have been stated in the preparation of chitosan nanoparticle which involves emulsification, emulsion droplet coalescence, reverse micellization and ionic gelation. ${ }^{5}$ Among these methods ionic gelation method is extensively used for because of its feasibility. ${ }^{5}$ Chitosan nanoparticles (CH-NP) have been explored for their antibacterial antifungal activity whereas, $\mathrm{CH}-\mathrm{NP}$ loaded with drug has been looked for therapeutic values in various neurological
Volume 3 Issue 2 - 2016

\author{
Pravin Potdar D, Aashutosh Shetti U \\ Department of Molecular Medicine \& Biology, Jaslok Hospital \& \\ Research Centre, India
}

\begin{abstract}
Correspondence: Pravin Potdar D, Department of Molecular Medicine \& Biology, Jaslok Hospital \& Research Centre, Maharashtra, India, Tel 912266573445, 919820833530 , Fax 912223520508,

Email ppotdar@jaslokhospital.net, ppravin0I2@gmail.com
\end{abstract}

Received: January 12, 2016 | Published: February 10, 2016

disorders, HIV and cancer. ${ }^{6,7}$ Use of chitosan in the drug delivery involves less Cytotoxicity, for the reason being, it is a biodegradable molecule which is degraded easily by the kidney in vivo. ${ }^{8}$

Many commercial anti-cancer drugs are available in the market and many drug discovery trials are being performed in the cancer research. It is evident that somewhere around traditional medicinal approach fails to provide beneficial effect and lacks the specificity and causes Cytotoxicity to the healthy cells. Development of drug resistance within the cancer cells and inability to penetrate these cancer cells are the reasons behind the failure of commercial medicine. The reason for adapting $\mathrm{CH}-\mathrm{NP}$ drug delivery system are, they are biocompatible, and cheaper. ${ }^{9}$ Due to their nano size, CH-NPs are internalized by the cells easily and hence they can deliver drug to cells more precisely. This increases the specificity of CH-NP and is the reason for their wide range of therapeutic importance. ${ }^{10}$ This also makes them potential vehicle system for gene delivery. CH-NPs have shown tremendous therapeutic significance in various types of cancer. ${ }^{11-13}$ $\mathrm{CH}-\mathrm{NP}$ affects the proliferation of human gastric cancer cell line by mechanisms yet to be discovered. ${ }^{12}$ In the breast cancer mice model, chitosan nanoparticles containing anti-Rho small interfering RNA have shown tumor anti-angiogenesis. ${ }^{13}$ Modified CH-NPs have been tried in the oral vaccination for breast cancer which resulted in improved immune protection in mice model. ${ }^{14}$ When developing a nanoparticle based drug delivery system, it is important to consider target specificity of nanoparticle which is lacked by the traditional medicinal approach. Lack of target specificity often results in the side effects. Many scientists have developed an effective modification in the Ch-Nps which help in achieving the goal of target specificity. The strategy mainly involves the binding of protein on to the nanoparticles for which the receptors are present exclusively on the tumor cells. The best suitable example for this involves binding of $\alpha v \beta 3$ integrin to the CH-NP. The receptor for this protein is widely expressed in various tumors and this strategy has shown positive effects in the ovarian cancer model. ${ }^{15}$ Another good example being the modification of CH-NP with the folic acid since cancer cell express the folic acid receptors on their cell surface. ${ }^{16}$ Dual encapsulation strategy has been employed where two drugs have been encapsulated within the chitosan. Encapsulation of paclitaxel and thymoquinone within a $\mathrm{CH}-$ NP resulted in effective breast cancer therapy. ${ }^{17} \mathrm{CH}-\mathrm{NP}$ increases the 
immune response by not only elevating the $\operatorname{IgG}, \operatorname{IgA}$ and $\operatorname{IgM}$ but also the IL-2, Il-4 and IL-6 as observed in murine model. ${ }^{4}$ As mentioned earlier the wide range of therapeutic significance is mainly due to the modification of CH-NP within the tumor microenvironment. Tumor microenvironment which has poor vasculature does cause increase in temperature and development of acidic condition. The amino group of the chitosan is protonated in acidic condition causes CH-NPs to swell, resulting in the faster drug release. ${ }^{18}$ In addition, the poor vasculature causes the accumulation of mancro-molecule in the tumor microenvironment. This phenomena was described as enhanced permeability and retention (EPR). ${ }^{19}$ Protonation in acid condition and EPR can be the main aspects for adapting CH-NP drug system in the treatment of cancers. Important thing to notice is use of chitosan is being carried out as a carrier of drug or nucleic acid and less significance is given to the therapeutic values of chitosan nanoparticle itself. The chitosan possesses the cytotoxic effect in cancer cells when compared to the normal cells. It induces apoptosis and causes cell cycle arrest in the oral cancer cells. ${ }^{20}$ Modifications in the chitosan molecule have been adapted in order to improve the biodegradability and immunological activity. The important example for this being the glycation of chitosan molecule. Glycated chitosan is predicted to be suitable for the incorporation in late-stage cancer where patients have very low tolerance towards the chemotherapy and radiation therapy. ${ }^{4}$ Also the glycol chitosan nanoparticle system have been used as the theranostic agent in the cancer where simultaneous diagnosis and therapy can be performed. ${ }^{21}$

The scope of CH-NP is wide open for many discoveries which involve identifying the signalling pathways altered by the CH-NP in the cancers. Also the studies can be further expanded for their effects on the cancer stem cells and circulating tumor cells. This is an important aspect since these two cell types are the main reason behind tumor metastasis and recurrence of cancer after drug treatment respectively. Overall chitosan is an important molecule in the cancer research and needs to be explored further and this may soon result in the groundbreaking discovery in the cancer treatment which will be beneficial for the thousands of patients suffering from various cancers.

\section{Acknowledgements}

Authors wish to acknowledge Management of Jaslok Hospital $\&$ Research Centre, Mumbai for giving an opportunity to do basic research in the field on nanotechnology applications in treatment of cancer.

\section{Conflict of interest}

The author declares no conflict of interest.

\section{References}

1. Seeta Rama Raju G, Benton L, Pavitra E, et al. Multifunctional Nanoparticles: Recent Progress in Cancer Therapeutics. Chem Commun (Camb). 2015;51(68):13248-13259.

2. Brigger I, Dubernet C, Couvreur P. Nanoparticles in cancer therapy and diagnosis. Adv Drug Deliv Rev. 2002;54(5):631-651.

3. Eliaz RE, Nir S, Marty C, et al. Determination and Modeling of Kinetics of Cancer Cell Killing by Doxorubicin and Doxorubicin Encapsulated in Targeted Liposomes. Cancer Res. 2004;64(2):711-718.
4. Li X, Min M, Du N, et al. Chitin, chitosan, and glycated chitosan regulate immune responses: the novel adjuvants for cancer vaccine. Clin Dev Immunol. 2013;2013:387023.

5. Grenha A. Chitosan nanoparticles: a survey of preparation methods. $J$ Drug Target. 2012;20(4):291-300.

6. Wilson B, Samanta MK, Muthu MS, et al. Design and evaluation of chitosan nanoparticles as novel drug carrier for the delivery of rivastigmine to treat Alzheimer's disease. Ther Deliv. 2011;2(5):599609 .

7. Dev N, Binulal S, Anitha S, et al. Preparation of poly(lactic acid)/chitosan nanoparticles for anti-HIV drug delivery applications. Carbohydrate Polymers. 2010;80(3):833-838.

8. Kean T, Thanou M. Biodegradation, biodistribution and toxicity of chitosan. Adv Drug Deliv Rev. 2010;62(1):3-11.

9. Grenha A, Grainger CI, Dailey LA, et al. Chitosan nanoparticles are compatible with respiratory epithelial cells in vitro. Eur J Pharm Sci. 2007;31(2):73-84.

10. Malatesta $\mathrm{M}$, Grecchi $\mathrm{S}$, Chiesa $\mathrm{E}$, et al. Internalized chitosan nanoparticles persist for long time in cultured cells. Eur J Histochem. 2015;59(1):2492.

11. Aruna U, Rajalakshmi R, Vinesha IMYV, et al. Role of Chitosan Nanoparticles in Cancer Therapy. Int J Innov Pharm Res. 2013;4(3):318324.

12. Qi LF, Xu ZR, Li Y, et al. In vitro effects of chitosan nanoparticles on proliferation of human gastric carcinoma cell line MGC803 cells. World J Gastroenterol. 2005;11(33):5136-5141.

13. Xu Y, Wen Z, Xu Z. Chitosan nanoparticles inhibit the growth of human hepatocellular carcinoma xenografts through an antiangiogenic mechanism. Anticancer Res. 2009;29(12):5103-5109.

14. Liu Z, Lv D, Liu S, et al. Alginic acid-coated chitosan nanoparticles loaded with legumain DNA vaccine: effect against breast cancer in mice. PLoS One. 2013;8(4):e60190.

15. Han HD, Mangala LS, Lee JW, et al. Targeted gene silencing using RGDlabeled chitosan nanoparticles. Clin Cancer Res. 2010;16(15):3910 3922 .

16. Song H, Su C, Cui W, et al. Folic acid-chitosan conjugated nanoparticles for improving tumor-targeted drug delivery. Biomed Res Int. 2013;2013:723158

17. Soni P, Kaur J, Tikoo K. Dual drug-loaded paclitaxel-thymoquinone nanoparticles for effective breast cancer therapy. J Nanoparticle Res. 2015;17(1):18.

18. Mateescu MA, Ispas-Szabo P, Assaad E. Controlled Drug Delivery: The Role of Self-Assembling Multi-Task Excipients. Elsevier Science; 2014.

19. Maeda H. The enhanced permeability and retention (EPR) Effect in tumor vasculature: The key role of tumor-selective macromolecular drug targeting. Adv Enzyme Regul. 2001;41:189-207.

20. Wimardhani YS, Suniarti DF, Freisleben HJ, et al. Chitosan exerts anticancer activity through induction of apoptosis and cell cycle arrest in oral cancer cells. J Oral Sci. 2014;56(2):119-126.

21. Rhee JK, Park OK, Lee A, et al. Glycol Chitosan-Based Fluorescent Theranostic Nanoagents for Cancer Therapy. Mar Drugs. 2014;12(12):6038-6057. 